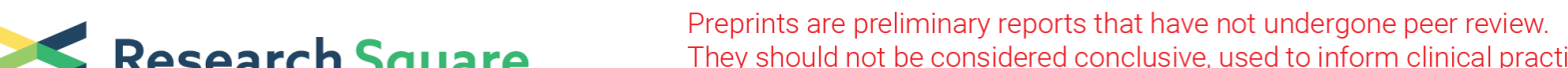 $\begin{array}{ll}\text { Research Square } & \text { They should not be considered conclusive, used to inform clinical practice, } \\ \text { or referenced by the media as validated information. }\end{array}$
}

\section{5-year Recurrence Prediction After Hepatocellular Carcinoma Resection Using Deep Learning and Cox Regression Models: A Large Prospective Study}

Hon-Yi Shi

Kaohsiung Medical University

King-The Lee

Park One International Hospital

Chong-Chi Chiu

I-Shou University College of Medicine

Jhi-Joung Wang

Chi Mei Medical Center

Ding-Ping Sun

Chi Mei Medical Center

Hao-Hsien Lee ( $\square$ Hao_Hsien@hotmail.com )

Chi Mei Hospital Liou Ying

\section{Research Article}

Keywords: hepatocellular carcinoma, resection, deep learning, deep neural network, recurrent neural network, Cox regression, recurrence, feature importance, surgeon volume, hospital volume

Posted Date: November 18th, 2021

DOI: https://doi.org/10.21203/rs.3.rs-1062761/v1

License: (c) (1) This work is licensed under a Creative Commons Attribution 4.0 International License.

Read Full License 


\section{Abstract}

\section{Background}

Risk of hepatocellular carcinoma (HCC) recurrence after surgical resection is unknown. Therefore, the aim of this study was 5-year recurrence prediction after $\mathrm{HCC}$ resection using deep learning and Cox regression models.

\section{Methods}

This study recruited $520 \mathrm{HCC}$ patients who had undergone surgical resection at three medical centers in southern Taiwan between April, 2011, and December, 2015. Two popular deep learning algorithms: a deep neural network (DNN) model and a recurrent neural network (RNN) model and a Cox proportional hazard $(\mathrm{CPH})$ regression model were designed to solve both classification problems and regression problems in predicting HCC recurrence. A feature importance analysis was also performed to identify confounding factors in the prediction of $\mathrm{HCC}$ recurrence in patients who had undergone resection.

Results

All performance indices for the DNN model were significantly higher than those for the RNN model and the traditional $\mathrm{CPH}$ model $(p<0.001)$. The most important confounding factor in 5 -year recurrence after $\mathrm{HCC}$ resection was surgeon volume followed by, in order of importance, hospital volume, preoperative Beck Depression Scale score, preoperative Beck Anxiety Scale score, co-residence with family, tumor stage, and tumor size.

\section{Conclusions}

The DNN model is useful for early baseline prediction of 5-year recurrence after $\mathrm{HCC}$ resection. Its prediction accuracy can be improved by further training with temporal data collected from treated patients. The feature importance analysis performed in this study to investigate model interpretability provided important insights into the potential use of deep learning models for predicting recurrence after $\mathrm{HCC}$ resection and for identifying predictors of recurrence.

\section{Introduction}

Hepatocellular carcinoma (HCC) remains the fourth leading cause of cancer-related death in the world. Liver resection is the mainstay of curative treatment for $\mathrm{HCC}$, but HCC recurrence after resection remains high; $60-70 \%$ of patients who undergo HCC resection develop recurrence within 5 years after surgery [1, 2]. In patients who have undergone HCC resection, recurrence is significantly associated with demographic attributes, aggressive tumor pathology, surgeon volume, hospital volume, and preoperative quality of life [1-3]. Therefore, accurately predicting recurrence after HCC resection is critical for effective delivery of precision medicine. The capability to predict recurrence in individual patients is also essential because it enables clinicians to gauge treatment outcomes. 
Recently developed deep-learning algorithms based on multilayer perceptrons use clinical pathological data to predict cancer surgery outcomes [4-6]. The utility of deep learning algorithms has been examined in various translational and clinical medicine settings. However, literature on their use for predicting cancer recurrence is relatively sparse [4-6]. Regarding recurrence analysis, deep-learning algorithms, which is a class of machine learning models, can use nonlinear risk functions for automatically modeling recurrence risk and for predicting individual medical outcomes based on learned representations. Recent advances in artificial intelligence, including deep learning algorithms, have also been applied in the medical field [4-8]. However, deep learning algorithms have yet to be used for predicting clinical prognosis after cancer surgery. The main reason is the high complexity of prediction algorithms relative to diagnostic algorithms, e.g., prediction algorithms require more numerous parameters. Therefore, the aim of this long-term prospective study was to integrate deep neural networks (DNNs) and recurrent neural networks (RNNs) in a deep learning algorithm for predicting 5-year recurrence of HCC in a large population of HCC patients who have undergone resection. Another aim was to compare the prediction performance of the DNN and the RNN deep learning models with that of a conventional Cox proportional hazard regression $(\mathrm{CPH})$ model.

\section{Patients And Methods}

\section{Subjects and study design}

The subjects of this study were HCC patients who had received surgical resection performed at one of three southern Taiwan medical centers between April, 2011, and December, 2015. Inclusion criteria were the following: (1) a histologic or a combined radiographic and laboratory diagnosis of HCC; (2) ability to communicate in Chinese or Taiwanese; and (3) agreement to participate in a questionnaire survey performed in the hospital ward. Major exclusion criteria included loss to follow up or death during the study period, which might have interfered with the analysis in this study. Figure 1 shows that, during the sample selection period, 819 subjects were eligible for participation. Of these, 299 were excluded due to loss to follow up or death. Therefore, 520 subjects participated in the preoperative (baseline) and postoperative 5-year assessments in this study. The institutional review boards at all participating hospitals approved the study protocol (KMUH-IRB-20110002). Additionally, all participants gave informed consent before they enrolled in this study.

\section{Instruments and potential predictors}

Research assistants collected demographic, clinical, and institutional data by reviewing questionnaire survey results and medical records. Data collection included gender, age, marital status, education, coresidence with the family, hepatitis $\mathrm{B}$, hepatitis $\mathrm{C}$, body mass index (BMI), Charlson comorbidity index (CCl), tumor size, tumor stage, American Society of Anesthesiologists score, average length of stay, 30day readmission after hospital discharge, surgeon volume, hospital volume, and preoperative Beck Anxiety Inventory (BAI) score and Beck Depression Inventory (BDI) score. The BAI and BDI were used to evaluate anxiety and depression in the HCC patients before surgery $[9,10]$. Each scale includes 21 self- 
evaluated items, each of which is scored from 1 to 4 point(s). A high BAl score or BDI indicates high severity of anxiety or depression, respectively. Thus, a high score before and after therapy suggests that the therapy was ineffective. The Chinese versions of the BDI and BAI used in this study have been validated and widely used in both clinical practice and research [11]. The surgeon volume and hospital volume data included in the dataset were sorted by total annual surgical procedures, and each procedure was assigned a unique identification code. In performance comparisons of the DNN, RNN, and CPH models, the above variables were the independent variables, and 5-year recurrence after HCC resection was the dependent variable.

\section{Development of the deep learning algorithms}

The neural network type most commonly used for real-world applications is the feedforward neural network, in which neurons are organized into distinct layers in order of topology. The first layer is the input layer, the last layer is the output layer, and the other layers are the hidden layers. This study defined a DNN as a feedforward network with more than one hidden layer. The DNN model was trained using back-propagation [12]. First, the transfer learning framework was used to train only the top layers that were randomly initialized by freezing all convolutional layers. The model was then trained using several epochs of data from our dataset, and validation loss was monitored by applying early stop criteria. The typical feature of the RNN architecture is a cyclic connection, which enables the RNN to update its current state based on past states and current input data [13]. Finally, grid-search method was used to optimize the hyperparameters and architectures of the deep learning DNN and RNN algorithms, and the numbers of epochs were determined by performing the tuning procedure described in supplementary Table 1.

\section{Statistical analysis}

The unit of analysis in this study was the individual patient with HCC after resection. The study characteristics were reported as mean \pm standard deviation (SD) and median interquartile range (IQR) for continuous variables and as frequency (\%) for categorical variables. Cox proportional hazards regression was performed to investigate hazard ratios (HRs) and $95 \%$ confidence intervals $(95 \% \mathrm{Cl})$ and to construct a model for predicting 5-year recurrence after $\mathrm{HCC}$ resection. Recurrence time was defined as the interval between the first HCC diagnosis and the first HCC recurrence.

Statistical analysis was performed in the following steps. In the first step, univariate Cox proportional hazards regression analyses were performed to identify significant $(p<0.05)$ predictors of 5 -year recurrence after $\mathrm{HCC}$ resection, which were then included in the forecasting models. In the second step, data for the study cohort of 520 subjects were randomly divided into three datasets: a training dataset containing data for 364 subjects was used for model development, a testing dataset containing data for 78 subjects was used for internal validation, and a validating dataset containing data for 78 subjects was used for external validation. The independent variables fitted to the forecasting models were significant predictors of 5-year recurrence after HCC resection, and the dependent variable was 5-year recurrence after $\mathrm{HCC}$ resection. After model training, model outputs were collected for each testing dataset. In the third step, 1,000 pairs of forecasting models with $95 \%$ confidence intervals were compared in terms of 
indices of performance in predicting 5-year recurrence after $\mathrm{HCC}$ resection. Independent t test was used to identify performance indices that significantly differed among the three models. Indices used for performance comparisons included accuracy, precision, recall, F-1 score, and area under the receiver operating characteristic (AUROC) curve. In the fourth and final step, permutation importance was calculated by using an algorithm to obtain an importance score for each confounder variable in the dataset [14]. Permutation importance was defined as the model score when the value of a single confounder was randomly changed. This technique has two advantages: first, it is model-agnostic; second, it can be performed repeatedly with different feature permutations.

The scikit-learn 0.21.2 implementation for Python (version 3.7.6; Python Software Foundation, Wilmington, Delaware, USA) was used to run the deep learning DNN and RNN models. The CPH model was computed with the Lifelines v0.22.2 implementation for Python v3.7.6 and double-checked with JMP10.0 (SAS Institute Inc., Cary, NC). All statistical tests were two-sided; a $p$ value less than 0.05 was considered statistically significant.

\section{Results}

\section{Study characteristics}

Table 1 shows that, during the study period, 5-year recurrence after $\mathrm{HCC}$ resection was $50.8 \%$. In patients with HCC recurrence after undergoing surgery, mean age was 61.4 years (SD, 10.7 years), median age was 62 years (IQR 55-69 years), and most (70.4\%) patients were male. The univariate analysis revealed that gender, age, marital status, education, co-residence with family, hepatitis $\mathrm{B}$, hepatitis $\mathrm{C}, \mathrm{BMI}, \mathrm{CCl}$, tumor size, tumor stage, American Society of Anesthesiologists score, average length of stay, 30-day readmission after hospital discharge, surgeon volume, hospital volume, and preoperative BAI and BDI scores are significantly $(p<0.05)$ associated with 5 -year recurrence after $\mathrm{HCC}$ resection (supplementary Table 2).

Table 1 Patient characteristics $(N=520)$ 


\begin{tabular}{|c|c|c|}
\hline Variables & $\begin{array}{l}\text { Mean } \pm \text { standard deviation/ } \\
\text { median [IQR] }\end{array}$ & $n(\%)$ \\
\hline \multicolumn{3}{|l|}{ Gender } \\
\hline Male & & $366(70.4 \%)$ \\
\hline Female & & $154(29.6 \%)$ \\
\hline Age, years & $61.4 \pm 10.7 / 62[55-69]$ & \\
\hline \multicolumn{3}{|l|}{ Marital status } \\
\hline Divorced or widowed & & $69(13.3 \%)$ \\
\hline Married & & $451(86.7 \%)$ \\
\hline Education, years & $12.5 \pm 4.9 / 12[9-16]$ & \\
\hline \multicolumn{3}{|l|}{ Co-residence with the family } \\
\hline Yes & & $456(87.7 \%)$ \\
\hline No & & $64(12.3 \%)$ \\
\hline \multicolumn{3}{|l|}{ Hepatitis B } \\
\hline Yes & & $163(31.3 \%)$ \\
\hline No & & $357(68.7 \%)$ \\
\hline \multicolumn{3}{|l|}{ Hepatitis C } \\
\hline Yes & & $99(19.0 \%)$ \\
\hline No & & $421(81.0 \%)$ \\
\hline Body mass index, $\mathrm{kg} / \mathrm{m}^{2}$ & $24.7 \pm 3.5 / 24.5[22.4-26.6]$ & \\
\hline Charlson Comorbidity Index, score & $1.2 \pm 1 / 1[0-2]$ & \\
\hline Tumor size, cm & $4.7 \pm 3.4 / 3.8[2.5-5.9]$ & \\
\hline \multicolumn{3}{|l|}{ Tumor stage } \\
\hline Stage I & & $250(48.1)$ \\
\hline Stage II & & 155(29.8) \\
\hline Stage III & & 73(14.0) \\
\hline Stage IV & & $42(8.1)$ \\
\hline \multicolumn{3}{|c|}{ American Society of Anesthesiologists, score } \\
\hline I & & $130(25.0 \%)$ \\
\hline
\end{tabular}




\begin{tabular}{|lll|}
\hline IIII & $197(37.9 \%)$ \\
\hline Average length of stay, days & $193(37.1 \%)$ \\
\hline 30 -day readmission after discharge & $10.9 \pm 7.9 / 9[7-11]$ & \\
\hline Yes & & $65(12.5 \%)$ \\
\hline No & & $455(87.5 \%)$ \\
\hline Surgeon volume, cases/year & $5.3 \pm 2.4 / 7[1-14]$ & \\
\hline Hospital volume, cases/year & $21.4 \pm 18.5 / 57[23-97]$ & \\
\hline Preoperative BDI score & $4.8 \pm 4 / 5[2-5]$ & $264(50.8 \%)$ \\
\hline Preoperative BAl score & $2 \pm 2.8 / 2[0-3]$ & $256(49.2 \%)$ \\
\hline 5-year recurrence after surgery & & \\
Yes & & \\
\hline No & & \\
\hline
\end{tabular}

$I Q R$ interquartile range, $B D /$ Beck Depression Inventory, $B A /$ Beck Anxiety Inventory.

\section{Comparison of forecasting models}

The study characteristics did not significantly differ between the training and testing datasets (supplementary Table 3); therefore, samples were compared between the training and testing datasets to increase reliability of the validation results. In Figure 2, the performance indices for the training dataset indicate that the DNN model had significantly higher accuracy, precision, recall, F1 score, and AUROC values compared to the RNN and CPH models $(p<0.001)$.

\section{Permutation importance of potential confounders in the DNN model}

Figure 3 shows the results of permutation importance of confounders in the DNN model. Surgeon volume and hospital volume were the most important confounders when predicting 5-year recurrence after HCC recurrence followed by preoperative BDI score, preoperative BAI score, co-residence with family, tumor stage, and tumor size.

\section{Sensitivity analysis}

Next, performance indices obtained for the testing dataset in internal validation and for the validating dataset in external validation were compared among the forecasting models (Table 2). Supplementary Table 3 shows that, for both the testing and validating datasets, the DNN model had better performance indices compared to the RNN and $\mathrm{CPH}$ models. 
Table 2

Comparison of performance indices between deep learning and Cox regression models used to predict 5-year recurrence after HCC resection.in the testing and validating datasets

\begin{tabular}{|lccccc|}
\hline Models & Accuracy & Precision & Recall & F1 score & AUROC \\
\hline Testing dataset $(n=78)$ & & & & & \\
\hline Deep neural network & $84.39 \%$ & $83.46 \%$ & $82.11 \%$ & $79.92 \%$ & 0.93 \\
\hline Recurrent neural network & $60.63 \%$ & $62.51 \%$ & $51.14 \%$ & $63.57 \%$ & 0.83 \\
\hline Cox regression & $53.57 \%$ & $50.84 \%$ & $34.78 \%$ & $40.64 \%$ & 0.52 \\
\hline Validating dataset $(n=78)$ & & & & & \\
\hline Deep neural network & $84.74 \%$ & $84.60 \%$ & $83.56 \%$ & $79.17 \%$ & 0.93 \\
\hline Recurrent neural network & $59.20 \%$ & $71.73 \%$ & $71.78 \%$ & $61.96 \%$ & 0.83 \\
\hline Cox regression & $50.73 \%$ & $68.36 \%$ & $39.23 \%$ & $49.50 \%$ & 0.54 \\
\hline HCC hepatocellular carcinoma, AUROC area under the receiver operating characteristic. \\
\hline
\end{tabular}

\section{Discussion}

To the best of our knowledge, this study is the first to use deep learning DNN and RNN models to investigate 5-year recurrence after $\mathrm{HCC}$ resection. Indices of performance in predicting 5-year recurrence after $\mathrm{HCC}$ resection were compared among the DNN, RNN, and CPH models. For a given set of demographic, clinical, and institutional inputs, the DNN model clearly had superior performance indices compared to the other two models. Notably, our long-term prospective study collected longitudinal registry data from three different medical institutions, which provided a real-world depiction of current surgical treatment for HCC patients. In contrast, previous works have used data from a single medical center [4-8]. Moreover, using registry data obtained from three medical centers mitigated the potential for referral bias or bias caused by analyzing the practices of a single surgeon or a single institution.

In this long-term prospective study, our analysis demonstrated that deep-learning DNN and RNN models are superior to conventional $\mathrm{CPH}$ model in predicting $\mathrm{HCC}$ recurrence after resection. A review of previous literature indicates that an increasing number of studies are integrating deep-learning models in analytic approaches used in oncologic research [8]. Most of these studies are related to either diagnostic work-up, such as radiographic image analysis and cytopathologic interpretation, or genomic/molecular analysis for biomarker discovery; to date, studies that have used deep-learning models for predicting recurrence in oncology patients remain limited.

For analyzing recurrence after HCC resection, DNN models have three main strengths. First, as discussed earlier, use of DNN models enables a superior fit to variables with nonlinear relationships, which is applicable when examining real-life variables. Notably, previous works have demonstrated that numerous clinical-laboratory variables are non-linearly associated with medical outcomes and have implied that deep-learning models may be more appropriate than linear regression models in terms of predicting risk 
of various medical outcomes [4-8]. Second, deep-learning models can automatically learn feature representations from raw clinical data without explicit feature engineering; additionally, nonlinear risk functions can be used for fitting deep-learning models to censored data for recurrence after resection [4, 6]. That is, deep-learning models excel at identifying nonlinear relationships among data, and they easily accommodate censoring of recurrence data after resection. Third, this study suggests that DNN and RNN models outperform the $\mathrm{CPH}$ model when large feature sets are used. Since a deep-learning model can learn feature representation, perhaps the most important strength of the deep-learning model is its capability to analyze large feature sets, which is particularly beneficial in biomedical research since including numerous variables in conventional linear regression models can cause overfitting.

In line with previous studies, treatment at a high-volume hospital or by a high volume surgeon was significantly associated with good medical outcomes [15-17]. A previous study of a nationwide population provided additional insight into the combined effects of surgeon volume and hospital volume on survival after hepatic resection. The authors concluded that treatment by a high-volume surgeon and treatment in a high-volume hospital were the two important predictors of short-term survival after hepatic resection [15]. Notably, they defined high-volume hospitals as those that performed more than 245 hepatic resection procedures annually and defined high-volume surgeons as those who performed more than 59 hepatic resection procedures annually. Another study reviewed proposed guidelines for hepatobiliary surgery and addressed the effects of volume on outcomes of hepatobiliary surgery. The authors recommended the establishment of an expert panel to draft preparatory and preliminary agreements on the clarification and standardization of technical terminology used to discuss HCC resection and indicators for $\mathrm{HCC}$ resection [16]. Lu et al. retrospectively analyzed 23,107 major hepatectomies for HCC patients and concluded that surgeon volume and hospital volume are significant independent predictors of postoperative recurrence $(p<0.001)$ [17]. The best explanation for this finding is that 'practice makes perfect', i.e., surgeons who perform a high volume of HCC resection procedures are the most likely to achieve a high level of surgical skill, which then reduces the postoperative rate of HCC recurrence in their patients. Likewise, hospitals that perform a high volume of HCC resection procedures have the lowest recurrence rates because they have the most opportunities and incentive to establish and implement an effective system for preventing recurrence throughout a given episode of inpatient care. Another possible explanation is that high volume hospitals have the most incentive and the most opportunities to develop standard procedures for care and after complex surgical procedures such as HCC resection.

Regarding use of both preoperative BDI score and preoperative BAI score as predictors of cancer recurrence, Wang et al performed a meta-analysis of patient data from a selection of 17 eligible cohort studies involving 282,203 breast cancer patients to investigate whether baseline preoperative BAI and BDI scores are prognostic predictors of cancer recurrence [18]. Their meta-analysis confirmed that depression, anxiety, and the combination of depression and anxiety are associated with recurrence in patients with breast cancer. Several factors may explain the increased recurrence of cancer in patients with depression and anxiety, including unhealthy lifestyle factors, treatment nonadherence, and biological mechanisms. A recent multicenter, cross-sectional study of 996 patients diagnosed with mixed tumors also showed that 
patients newly diagnosed with cancer tend to have high anxiety about cancer recurrence [19]. The study also found that fear of cancer recurrence was significantly associated with financial burden, receipt of chemotherapy, severe illness in childhood, and emotional disturbances (anxiety and depression). In Chen et al., a nationwide population-based cohort study was performed to investigate the association between depressive disorders and tumor recurrence risk in patients who had received curative surgery for breast cancer [20]. The cohort with depressive disorder had a significantly higher risk of postoperative recurrence compared to the cohort without depressive disorder ( $\mathrm{HR}=1.373,95 \% \mathrm{Cl} 1.09 \sim 1.72, p=0.005)$. A possible explanation is that impaired endocrine function and impaired hypothalamic-pituitary-adrenal function in patients with depressive disorder may contribute to increased risk of recurrence after breast cancer surgery.

Su et al. administered the Mini International Neuropsychiatric Interview in breast cancer patients to investigate whether family support is associated with major depressive disorder [21]. The study found that family support, which has a well-established positive association with breast cancer outcome, also has a negative association with depression risk. Another prospective 23-year study of 373 subjects similarly reported that, compared to patients with weak family support, those who had strong family support tended to have lower severity of depression at baseline and more rapid improvement in depression [22]. Family caregivers may be ill prepared to assume responsibilities such as providing family members with information on the disease and treatment or providing instruction in technical and care skills [23]. Moreover, caregiving by family members must be balanced against already established roles and role responsibilities. Additionally, family members who assume a caregiving role may have their own emotional responses to the diagnosis and prognosis of the patient and may require coaching and emotional support. As previously noted, however, further studies are needed to explore the influence of family support on HCC recurrence after resection.

In agreement with previous works [24, 25], the present study found that advanced cancer stage was significantly associated with recurrence within 5 years after $\mathrm{HCC}$ resection. The patients analyzed in this study included 250 patients (48.1\%) in tumor stage I, 155 (29.8\%) in tumor stage II, 73 (14.0\%) in tumor stage III, and $42(8.1 \%)$ in tumor stage IV. Early diagnosis of HCC and curative retreatment are likely to help prevent recurrence. After surgery, HCC patients are often burdened by multiple cancer-related comorbidities that increase their risk of poor postoperative outcomes, including complications, a long hospital stay, a short survival time, and high treatment costs. Zhang et al. reported that, after curative resection, independent predictors of HCC recurrence included tumor size ( $>5 \mathrm{~cm}$ vs. $<5 \mathrm{~cm}$ ) (odds ratio, $\mathrm{OR}=1.45,95 \% \mathrm{Cl} 1.05-2.02)$ and tumor-node-metastasis (TNM) stage (III-IV vs. I-II) (OR=1.36, 95\% Cl 1.021.81) [24]. Kumar et al also pointed out that T-stage and increased tumor size were significantly associated with early postoperative recurrence $(p<0.001)$ [25].

This large, long-term, and prospective observational cohort study of HCC patients in Taiwan analyzed data from patients treated at three medical centers. The accuracy of the deep-learning DNN model developed in this study was superior to that of the RNN and CPH models in predicting 5-year recurrence after HCC resection. Three implications of this study are noted. First, the proposed DNN model may be 
useful for guiding the clinical care of HCC patients after resection. Second, healthcare administrators and managers at medical institutions should facilitate the prompt establishment of surgical teams designed to meet the specific needs of individual patients. Third, the Taiwan National Health Insurance Administration should include both surgical volumes and emotional outcomes in its guidelines for clinical treatment of HCC in order to achieve a broad nationwide improvement in care for these patients. However, further studies are needed to confirm the clinical relevance of the proposed DNN model in terms of its efficacy in predicting prognosis and optimizing medical management for HCC patients after resection.

This study has several limitations inherent in a large database analysis. First, although we likely examined one of the largest sample sizes among studies of this nature, the relatively small total number of patients in this study limited the reliability of the results obtained by the deep-learning models. Second, the analysis was limited to 5-year recurrence after HCC resection, which reduced the subset of HCC patients with surgery in which the DNN model is clinically applicable. Third, this study only compared DNN, RNN and CPH models. Further studies are needed to compare other forecasting models. Finally, this study did not compare other outcomes, e.g., patient-reported quality of life, because the database did not contain these outcome data. Nevertheless, the results can still be considered valid given the robustness and statistical significance of the results.

In conclusion, due to high recurrence rates in patients with HCC, accurate prediction of disease recurrence is of major importance for effectively tailoring adjuvant treatment and follow-up regimes for individual patients. The proposed deep-learning DNN model appears promising for use in prediction and stratification of 5-year recurrence risk after $\mathrm{HCC}$ resection based on clinical data. Predictors of postoperative 5-year recurrence can be discussed when educating $\mathrm{HCC}$ candidates in the expected course of recovery and health outcomes. Although the practical applicability of database studies such as this have been convincingly demonstrated in the literature, future studies can expand the range of clinical variables included in the analysis, which could obtain additional novel results and potentially improve prediction accuracy. Further experiments using the proposed DNN model would clarify its potential uses for developing, promoting, and improving health policies for treating HCC patients after surgery.

\section{Declarations}

Data availability: The data that support the findings of this study are available from the corresponding author, upon reasonable request.

Animal research: This was not an animal research.

Consent to participate: All patients gave written informed consent.

Consent to publish: All authors approved the manuscript for publication.

Plant reproducibility: None. 
Clinical trials registration: Not applicable.

Author contributions: HYS and HHL had full access to all the data in the study and take responsibility for the integrity of the data and the accuracy of the data analysis. Concept and design: HYS and HHL. Administrative support: HYS and HHL. Provision of study materials or patients: KTL, CCC, JJW, and DPS. Collection and assembly of data: HYS and HHL. Data analysis and interpretation: HYS and HHL. Manuscript writing: All authors. Final approval of manuscript: All authors. Obtained funding: HYS and HHL.

Funding: This work was financially supported by grants from Chi Mei Medical Center, Liouying (CLFHR 10931) and from Ministry of Science and Technology (MOST 108-2410-H-037-006-SS3) in Taiwan.

Conflict of interest: None of the authors report any conflicts of interest.

Ethical approval: The institutional review boards at all participating hospitals approved the study protocol (KMUH-IRB-20110002).

\section{References}

1. Chew XH, Sultana R, Mathew EN, et al. Real-World Data on Clinical Outcomes of Patients with Liver Cancer: A Prospective Validation of the National Cancer Centre Singapore Consensus Guidelines for the Management of Hepatocellular Carcinoma. Liver Cancer 2021;10:224-39.

2. Zhang $X, B a i, Y, X u L$, et al. Clinical and morpho-molecular classifiers for prediction of hepatocellular carcinoma prognosis and recurrence after surgical resection. Hepatol Int 2019;13:715-725.

3. Xu XF, Xing H, Han J, et al. Risk Factors, Patterns, and Outcomes of Late Recurrence After Liver Resection for Hepatocellular Carcinoma: A Multicenter Study From China. JAMA Surg 2019;154:209-217.

4. Nam JY, Lee JH, Bae J, et al. Novel Model to Predict HCC Recurrence after Liver Transplantation Obtained Using Deep Learning: A Multicenter Study. Cancers (Basel) 2020;12:2791.

5. Ji GW, Zhu FP, Xu Q, et al. Machine-learning analysis of contrast-enhanced CT radiomics predicts recurrence of hepatocellular carcinoma after resection: A multi-institutional study. EBioMedicine 2019;50:156-165.

6. Nagendran $M$, Chen $Y$, Lovejoy $C A$, et al. Artificial intelligence versus clinicians: systematic review of design, reporting standards, and claims of deep learning studies. BMJ 2020;368:m689.

7. Izci H, Tambuyzer T, Tuand K, et al. A Systematic Review of Estimating Breast Cancer Recurrence at the Population Level With Administrative Data. J Natl Cancer Inst 2020;112:979-988.

8. Xiang K, Jiang B, Shang D. The overview of the deep learning integrated into the medical imaging of liver: a review. Hepatol Int 2021;15:868-880. 
9. Beck AT, Steer RA. Beck Anxiety Inventory Manual. San Antonio, TX:

Psychological Corporation, 1993.

10. Beck AT, Steer RA, Brown G. Beck Depression Inventory (BDI-II) Manual. San Antonio, TX: Psychological Corporation, Harcourt Brace, 1996.

11. Lee HH, Chiu CC, Lin JJ, et al. Impact of preoperative anxiety and depression on quality of life before and after resection of hepatocellular carcinoma. J Affect Disord 2019;246:361-367.

12. LeCun Y, Bengio Y, Hinton G. Deep learning. Nature 2015;521:436-444.

13. Yu Y, Si X, Hu C, et al. A Review of Recurrent Neural Networks: LSTM Cells and Network Architectures. Neural Comput 2019;31:1235-1270.

14. Altmann A, Toloşi L, Sander O, et al. Permutation importance: a corrected feature importance measure. Bioinformatics 2010;26:1340-1347.

15. Chang CM, Yin WY, Wei CK, et al. The combined effects of hospital and surgeon volume on short-term survival after hepatic resection in a population-based study. PLoS One 2014;9:e86444.

16. Franchi E, Donadon M, Torzilli G. Effects of volume on outcome in hepatobiliary surgery: a review with guidelines proposal. Glob Health Med 2020;2:292-7.

17. Lu CC, Chiu CC, Wang JJ, et al. Volume-outcome associations after major hepatectomy for hepatocellular carcinoma: a nationwide Taiwan study. J Gastrointest Surg 2014;18:1138-1145.

18. Wang $X$, Wang N, Zhong $L$, et al. Prognostic value of depression and anxiety on breast cancer recurrence and mortality: a systematic review and meta-analysis of 282,203 patients. Mol Psychiatry 2020;25:3186-3197.

19. Luo X, Li W, Yang Y, et al. High Fear of Cancer Recurrence in Chinese Newly Diagnosed Cancer Patients. Front Psychol 2020;11:1287.

20. Chen SJ, Chang $\mathrm{CH}$, Chen $\mathrm{HC}$, et al. Association between depressive disorders and risk of breast cancer recurrence after curative surgery. Medicine (Baltimore) 2016;95:e4547.

21. Su JA, Yeh SC, Chang CC, et al. Depression and family support in breast cancer patients. Neuropsychiatr Dis Treat 2017;13:2389-2396.

22. Kamen C, Cosgrove V, McKellar J, Cronkite R, Moos R. Family support and depressive symptoms: a 23-year follow-up. J Clin Psychol 2011;67:215-223.

23. Given BA, Given CW, Kozachik S. Family support in advanced cancer. CA Cancer J Clin 2001;51:213231. 
24. Zhang Y, Chen SW, Liu LL, et al. A model combining TNM stage and tumor size shows utility in predicting recurrence among patients with hepatocellular carcinoma after resection. Cancer Manag Res 2018;10:3707-3715.

25. Kumar AM, Fredman ET, Coppa C, et al. Patterns of cancer recurrence in localized resected hepatocellular carcinoma. Hepatobiliary Pancreat Dis Int 2015;14:269-275.

\section{Figures}




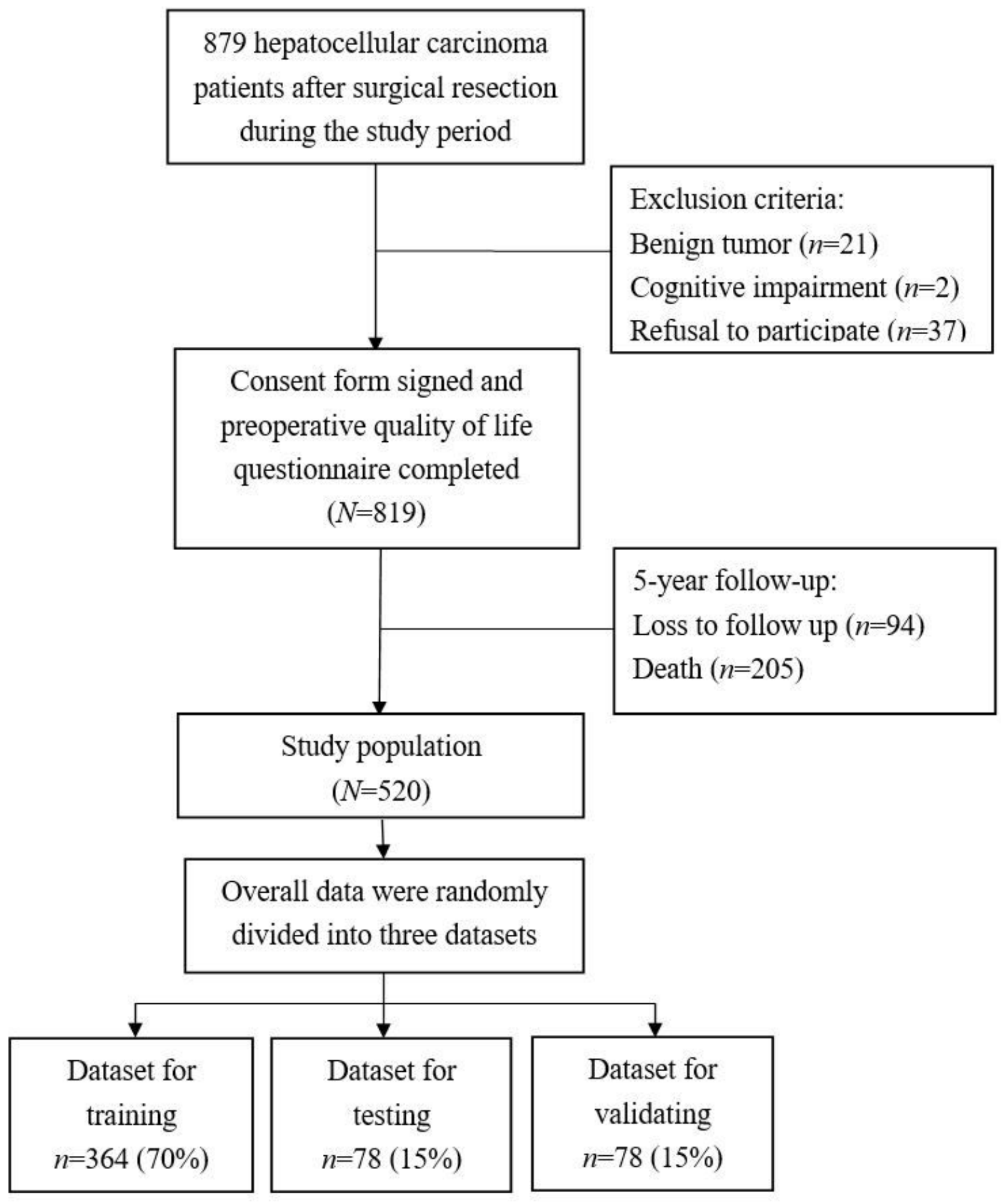

Figure 1

Flowchart of the study procedure. 

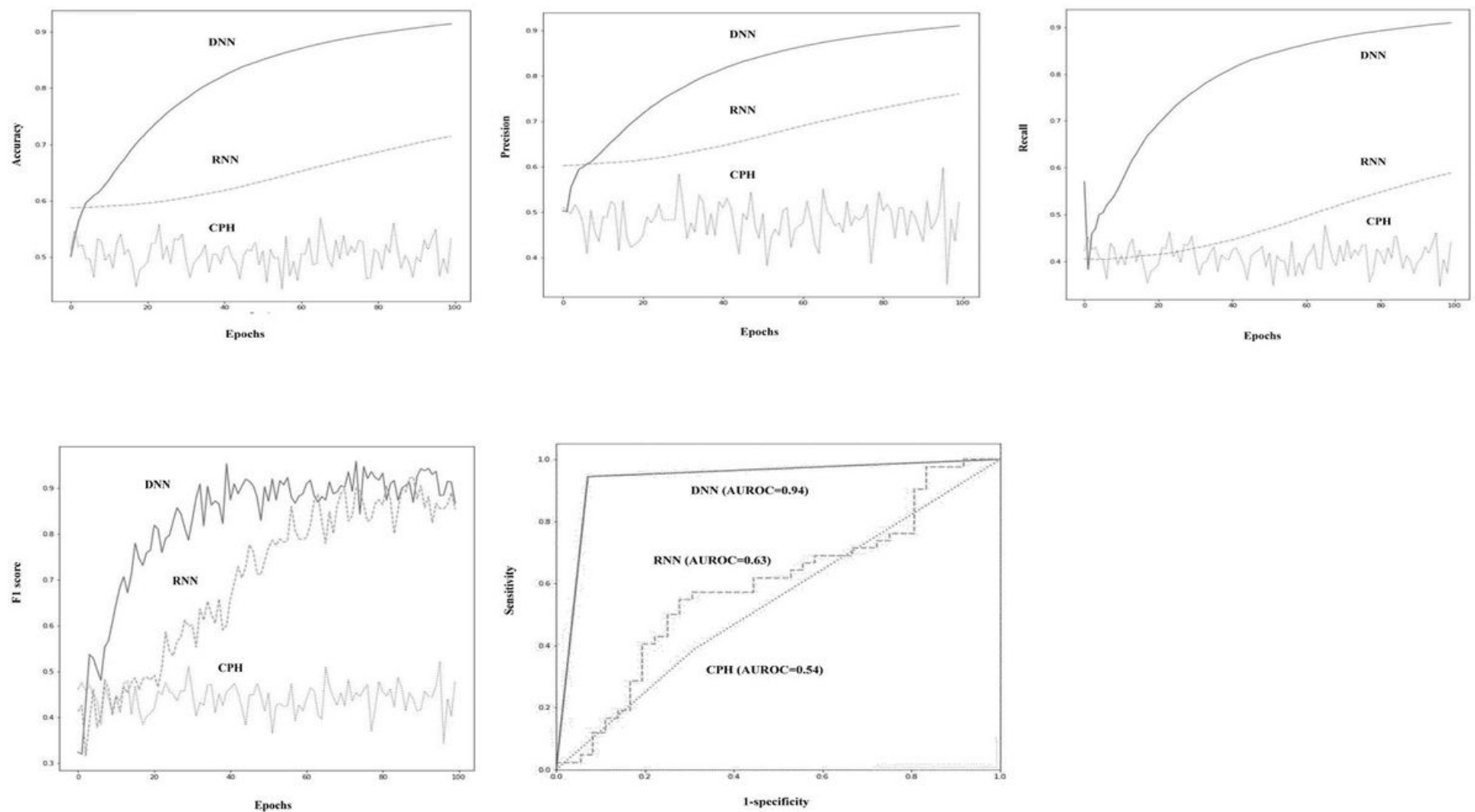

Figure 2

Comparison of accuracy, precision, recall, $\mathrm{F} 1$ score, and area under the receiver operating characteristics (AUROC) curve among the deep neural network (DNN), recurrent neural network (RNN), and Cox proportional hazard regression $(\mathrm{CPH})$ models for predicting 5-year recurrence after hepatocellular carcinoma $(\mathrm{HCC})$ resection in the training dataset $(n=364)$.

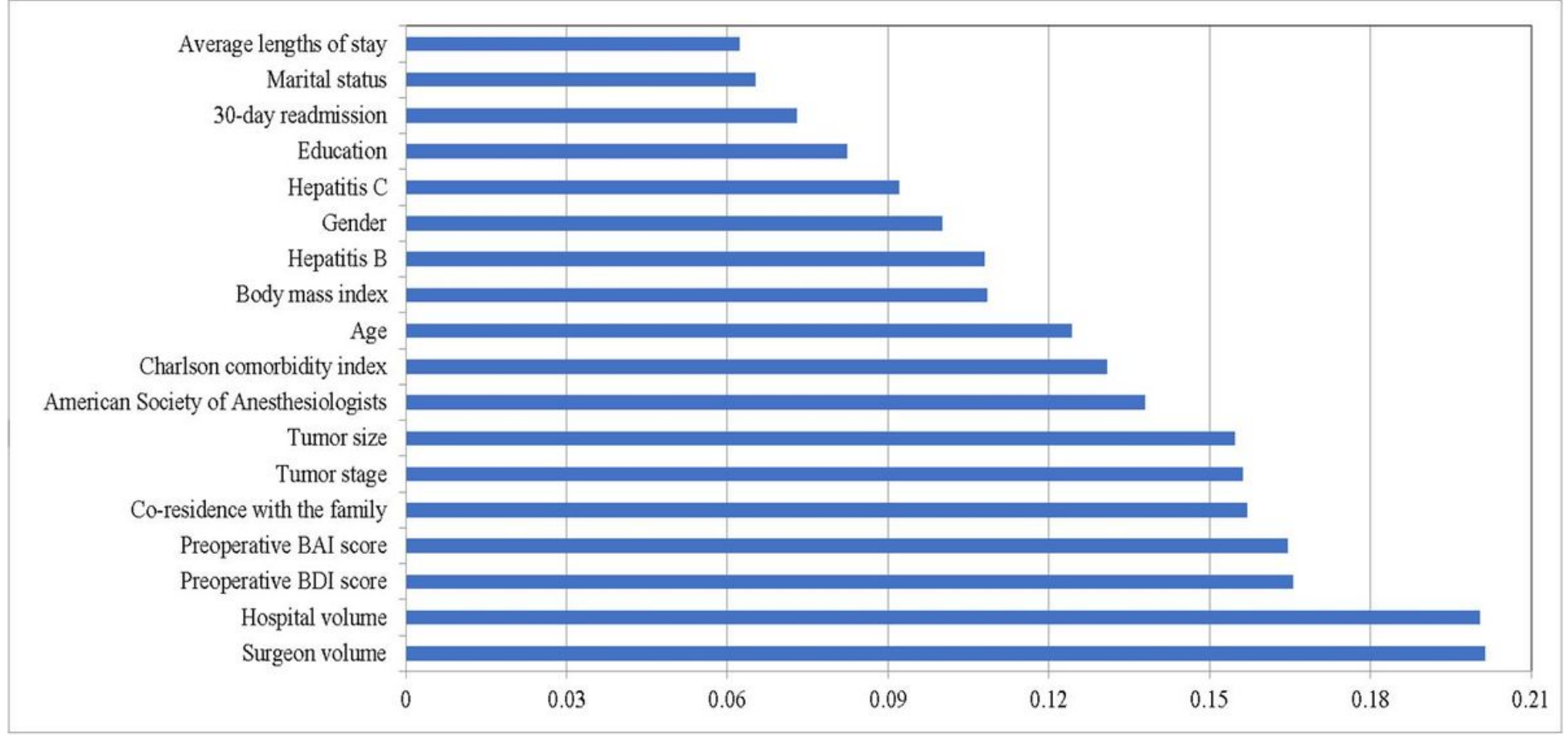


Figure 3

Permutation importance of the potential confounders in the deep neural network (DNN) model $(n=364)$.

\section{Supplementary Files}

This is a list of supplementary files associated with this preprint. Click to download.

- SupplementaryTable11.doc

- SupplementaryTable21.doc

- SupplementaryTable31.doc 